\title{
OPTIMAL LINEAR ARRAYS FOR WIDE BANDWIDTHS
}

\author{
R. G. MARSON \\ University of Sydney \& CSIRO Division of Radiophysics
}

\section{Introduction}

New technology has enabled modern aperture synthesis instruments to employ increasingly larger bandwidths. Large bandwidths can be exploited to enable a more complete coverage in the $(u, v)$ plane and hence aid image reconstruction. This relies on the source having a structure that is invariant with observing frequency or a suitable image reconstruction algorithm to take frequency variations into account.

Arrays that are ideal for this have baselines that scale geometrically. In this paper I describe a number of one dimensional arrays that meet or approach this. Throughout this discussion the instantaneous $(u, v)$ coverage is one dimensional and it is tacitly assumed that a two dimensional coverage is obtained either through earth rotation (with East-West baselines) or by rotating the array (as is possible in non-redundant masking). The effect of the wide bandwidth is to broaden each sample point radially. It is also assumed that the correlator has enough spectral channels to sample this broadened visibility point adequately.

\section{Ideal Arrays}

Ideal Arrays are arrays with baselines that exactly follow the rule:

$$
B_{n}=B_{1} \cdot\left[\frac{f_{\max }}{f_{\min }}\right]^{n-1}=B_{1} \cdot \alpha^{n-1} \quad n=1 \ldots M
$$

where $B_{n}$ is the $n^{\text {th }}$ shortest baseline and $f_{\min , \max }$ are the frequencies at the edges of the observing bandwidth. These arrays sample the $(u, v)$ plane completely with no overlap from a minimum radius $\left(\propto B_{1} f_{\min }\right)$ to an outer radius $\left(\propto B_{M} f_{\max }\right)$. The scaling factor $\alpha$ is related to the fractional bandwidth by $f_{B}=\frac{f_{\max }-f_{\min }}{f_{\max }}=\frac{\alpha-1}{\alpha}$

In the limiting case of $\alpha=1$ (ie. narrow bandwidth) no smearing of the visibility points will occur and construction of ideal arrays is impossible. Similarly if $\alpha$ is large (ie. $>2$ ) then the visibility points from different baselines will be broadened so much that a significant amount of overlap must occur. Only at intermediate values of $\alpha$ can ideal arrays exist. An exhaustive search for arrays with less than 6 elements reveals there are only two ideal arrays. They are shown in Figure 1.

The search technique can be split into two parts.

1. Initially for an $N$ element array the baselines in the array are ordered from shortest to longest and assigned to pairs of elements on the array. The number of possible orderings increases rapidly $\left(\propto N^{2} !\right)$ as the number of elements increases and is the reason this study did not consider arrays with more than 6 elements.

2. For each array ordering a set of equations can be written that an ideal array must satisfy. For example for the 4 element ideal array we have

$$
\alpha^{3}=1+\alpha
$$




\begin{tabular}{|c|c|c|c|c|c|}
\hline $\mathrm{N}$ & \multicolumn{3}{|c|}{ Array Ordering } & $\alpha$ & $f_{B}$ \\
\hline 3 & & 1 & $\alpha$ & 1.62 & $38 \%$ \\
\hline 4 & 1 & & $\alpha^{2}$ & 1.32 & $25 \%$ \\
\hline
\end{tabular}

Fig. 1. The two ideal arrays

$$
\begin{aligned}
& \alpha^{4}=\alpha+\alpha^{2} \\
& \alpha^{5}=1+\alpha+\alpha^{2}
\end{aligned}
$$

If there is a unique solution to these equations then it describes an ideal array.

Note: we arbitrarily set the minimum baseline length to 1 .

These arrays are ideal for a fixed value of $\alpha$ only. They also incorporate a rather large "hole" in the center of the $(u, v)$ plane. For both these reasons the optimal arrays described in the next section are preferable.

\section{Optimal Arrays}

If we give up the idea of completely sampling the $(u, v)$ plane and allow gaps and overlaps in the coverage what is the best array for an arbitrary number of elements and fractional bandwidth? To answer this question requires an error criterion and I chose to minimise

$$
\varepsilon=\sum_{i=1}^{N(N-1) / 2} H_{i}^{2} \quad \text { where } H_{i}=B_{i} f_{\min }-B_{i-1} f_{\max }
$$

$H_{i}$ is the size of the hole between the edge of one track and the beginning of the next. Baseline 0 is defined as being at the origin. This criterion penalises overlaps as much as holes in the coverage and also penalises a large central hole.

For each array ordering it is possible to explicitly write out an expression for $\varepsilon$. Using a symbolic algebra package we can solve for the array element positions while maintaining $\alpha$ as a free parameter. The ordering which produces the minimum error for a given value of $\alpha$ is called the optimum array. Shown in figure 2 are the optimum arrays for a practical range of fractional bandwidths. Some things to note are:

- No single ordering (except for the 3 element array which has only one ordering) is optimal for all values of $\alpha$. The discontinuities in the element positions are real and correspond to a point where one ordering of baselines becomes more favourable than the previous

- The 3 and 4 element arrays generated for $f_{B}=0$ are the well known nonredundant regularly spaced arrays

- A modest amount of fractional bandwidth can go a long way to filling the gaps 

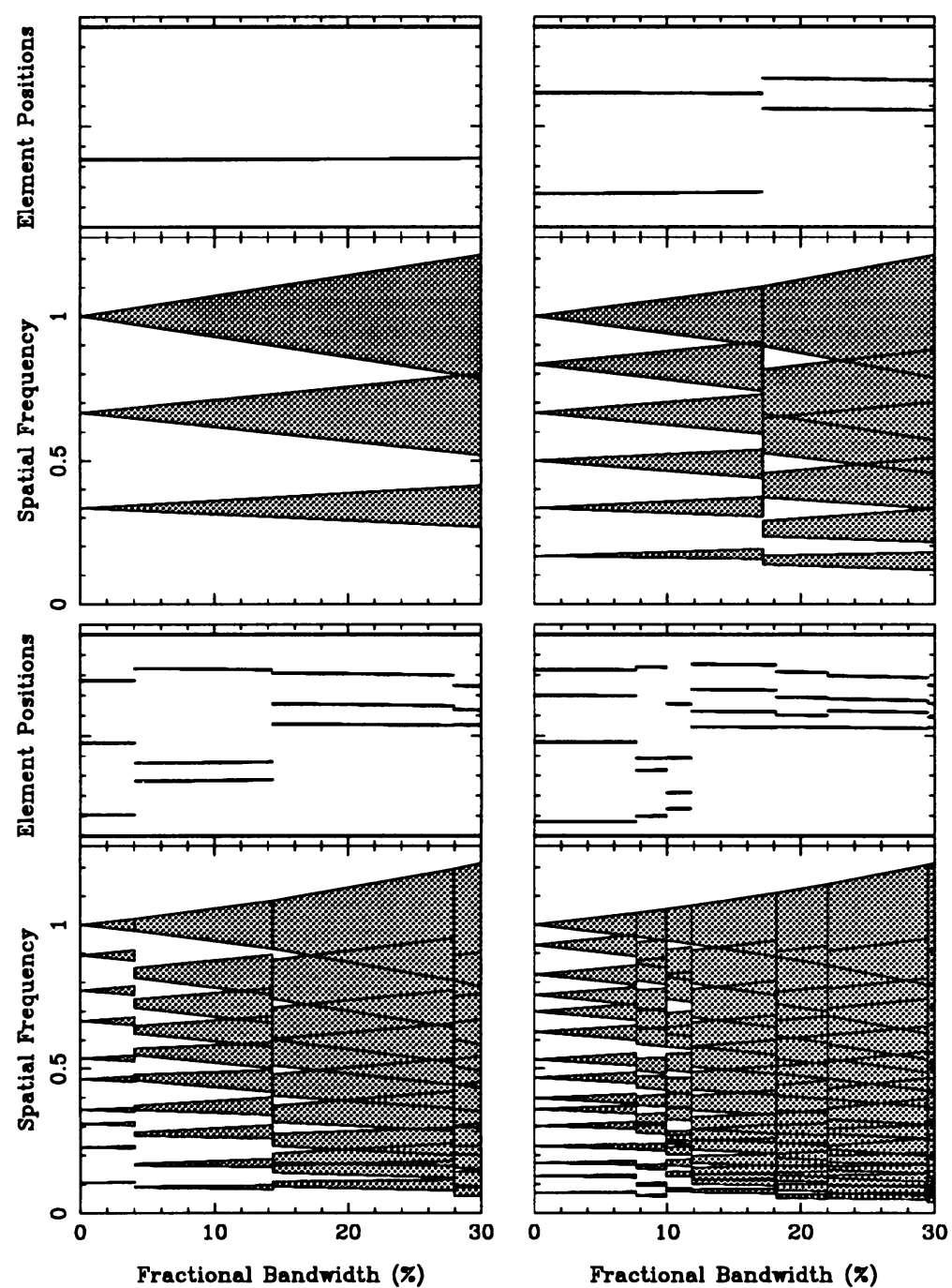

Fig. 2. Plot of element locations and spatial frequency coverage for the optimal arrays with 3-6 elements.

\section{Conclusion}

These array designs while limited in scope fill a small but important niche in synthesis imaging. In particular they are being used in the MAPPIT optical interferometer where photon noise restricts the number of array elements to a small value. 